\title{
Passive tumor targeting and imaging by using mercaptosuccinic acid-coated near-infrared quantum dots
}

\author{
Guimiao Lin' \\ Xiaomei Wang' \\ Feng Yin ${ }^{2}$ \\ Ken-Tye Yong ${ }^{2}$
}

'The Engineering Lab of Synthetic Biology and Research Institute of Uropoiesis and Reproduction, School of Medicine, Shenzhen University, Shenzhen, People's Republic of China; ${ }^{2}$ School of Electrical and Electronic Engineering, Nanyang Technological University, Singapore, Singapore
Correspondence: Ken-Tye Yong School of Electrical and Electronic Engineering, Nanyang Technological University, Block S2.2, Level B2, Nanyang Link, Singapore 639798, Singapore Email ktyong@ntu.edu.sg
This article was published in the following Dove Press journal:

International Journal of Nanomedicine

6 January 2015

Number of times this article has been viewed
Abstract: In this paper, we demonstrate the preparation of monodispersed quantum dots (QDs) as near-infrared (NIR) optical probes for in vivo pancreatic cancer targeting and imaging. The design of these luminescent probes involves functionalizing NIR QDs with ligand mercaptosuccinic acid (MSA), which targets the tumor site by enhanced permeability and retention effect. The colloidal and optical stability of the QDs can be maintained for $>1$ week. In vivo optical imaging studies in nude mice bearing pancreatic tumor show that the probes accumulate at tumor sites for $>2.5$ hours following intravenous injection of the functionalized NIR QDs. Tumor-labeling studies showed no evidence of harmful effects on the treated animals, even at a dose as high as $\sim 50 \mathrm{mg} / \mathrm{kg}$. These results demonstrate that the engineered MSA-functionalized QDs can serve as a diagnostic platform for early detection of cancer, as well as in image-guided precise surgical resection of tumors.

Keywords: QDs, near-infrared, mercaptosuccinic acid, pancreatic cancer, tumor targeting

\section{Introduction}

Semiconductor nanocrystals, also known as quantum dots (QDs), are highly luminescent nanoparticles with sizes ranging from $2 \mathrm{~nm}$ to $15 \mathrm{~nm} \cdot{ }^{1,2}$ QDs are composed of hundreds to thousands of atoms that commonly belong to groups II-VI (eg, $\mathrm{CdSe}$ and $\mathrm{CdTe}$ ), groups III-V (eg, InP), groups IV-VI (eg, PbS and PbSe), or group VI (eg, $\mathrm{Si}) .{ }^{3,4} \mathrm{QDs}$ have several unique optical properties far superior to those of the organic chromophores..$^{5-8}$ For example, QDs have high molar extinction coefficients, broad absorption bands, high quantum efficiency $(>50 \%)$, narrow emission spectra with full width at half-maximum $<50 \mathrm{~nm}$, high resistance to photobleaching, and higher excited state lifetimes. ${ }^{9,10}$ In addition to these features, it was demonstrated that QDs are at least 15 times brighter than organic dyes using the same excitation conditions. ${ }^{11}$ These unique optical properties can be utilized to enhance the signal-to-background ratio during microscopy imaging. ${ }^{12-14}$ Moreover, the QDs emission can be systematically tuned to emit from the visible to near-infrared (NIR) spectral region by simply manipulating their size, shape, composition, and structure. ${ }^{15-18}$ This optical tunability of QDs facilitates their use in multiplexed and real-time imaging. ${ }^{19,20}$ It was also reported that QDs can be used as a single probe for optical tracking studies in vitro, over a few hours using either laser scanning confocal microscopy or total internal reflection microscopy. ${ }^{21}$ In addition, QDs are potential candidates for two-photon imaging because these particles have a relatively large absorption cross section when compared to some organic dyes. ${ }^{22}$ Besides the unimodal imaging capability of functional QDs, other novel 
contrast agents can be incorporated into QD formulation for multimodal imaging. ${ }^{23}$

NIR in vivo imaging offers an exciting and powerful platform for many areas, ranging from in vitro molecular imaging to cancer diagnostics. ${ }^{24-26}$ In general, in vivo luminescence imaging with targeted QD probes requires deep penetration of light in and out of biological tissues. ${ }^{27}$ The absorption and scattering of the tissue and the absorbance of water are the main factors that limit the penetration depth of light. ${ }^{18}$ It was consistently reported that the best light penetration through tissues is achieved by using NIR wavelength light source, between $700 \mathrm{~nm}$ and $950 \mathrm{~nm} .{ }^{18}$ In addition to light penetration, significant background signals can be reduced upon using the NIR imaging technique. ${ }^{28,29}$ Therefore, NIR QDs can serve as a promising optical probe for improving the sensitivity of in vivo imaging. The illustration of functional, biocompatible, high-quantum yield (QY), and photostable NIR QDs will be a crucial step in the advancement of successful in vivo luminescence imaging for biomedical diagnostics.

QDs are mostly prepared in organic phase; therefore, their surfaces are functionalized with hydrophobic moieties to make them undispersible in biological fluids. ${ }^{30,31}$ More importantly, the hydrophobic moieties such as TOPO, oleic acid, and oleylamine will result in cytotoxicity to the biological environment, limiting their use in biological research such as cancer detection and therapy. ${ }^{32-34}$ Dozens of papers have reported novel surface functionalization strategies for QD nanoparticles to overcome this limitation. The most common approach so far has been to functionalize QD surface with short-chain thiolated surfactants, via the ligand exchange process. These thiolated surfactants are mercaptoacetic acid (MAA), thioglycerol, mercaptopyruvic acid, sodium 3-mercapto-1-propanesulfonate, mercaptopropionic acid, etc. ${ }^{35,36}$ However, it was observed that QD surface modification with some of these surfactants will cause a decrease in QD quantum efficiency and photostability as well as trigger the breakdown of QDs. ${ }^{37}$ Moreover, some of these surfactants are toxic by nature and not suitable for in vitro and in vivo studies. ${ }^{38-40}$ Thus, the main challenge in preparing stable aqueous dispersion of functionalized QDs for medical imaging involves the selection of small-molecular weight and low-toxicity thiolated ligands that are able to replace the hydrophobic surfactants on the QDs surface. ${ }^{41,42}$ Choosing the appropriate ligands will not only improve the QDs colloidal stability but also allow the nanoparticles to be "small" enough to excrete from body. It is well documented that surface functionalization chemistry of nanoparticles plays a crucial role in the development of diagnostic and therapeutic probes. For example, Choi et al reported the use of $\mathrm{CdSe} / \mathrm{CdS} / \mathrm{ZnS}$ QDs as a model system to evaluate the hydrodynamic diameter and surface charge conditions that allow rapid body excretion. ${ }^{42}$ They found that the excretion rate of nanoparticles is strongly dependent on their hydrodynamic diameter and the type of ligands used for surface coating. On the other hand, Qian et al have reported the preparation of polyethylene glycol (PEG)ylated SurfaceEnhanced Raman Scattering (SERS) gold nanoparticles with $80-90 \mathrm{~nm}$ size for in vivo tumor targeting and imaging. ${ }^{43}$ The authors have found that upon careful functionalization, the $\sim 80 \mathrm{~nm}$ gold nanoparticles can be effectively used as probes to detect cancerous areas, without observing any obstacles in terms of targeting and delivery. Gao et al have reported the preparation of $\sim 15 \mathrm{~nm}$ bioconjugated $\mathrm{CdSe} / \mathrm{ZnS}$ QDs and used them as optical probes for imaging tumor in nude mice. ${ }^{44} \mathrm{Cai}$ et al have demonstrated that RGD peptide-labeled CdTe/ZnS QDs with size of $\sim 20 \mathrm{~nm}$ can be used as NIR optical probes for the in vivo targeting and imaging of tumor vasculature. ${ }^{45}$ All these reports have demonstrated the importance of decorating the nanoparticles with appropriate ligands or polymers in order for them to be successfully used as bio-inert novel image contrast agents for detecting and mapping the location of cancerous areas.

In the present work, we report the engineering of mercaptosuccinic acid (MSA)-functionalized $\mathrm{CdTe}_{x} \mathrm{Se}_{1-x} / \mathrm{CdS}$ core-shell QDs as optical probes for pancreatic tumor targeting and imaging in live animals. The $\mathrm{CdTe}_{x} \mathrm{Se}_{1-x} / \mathrm{CdS}$ QDs were prepared by a straightforward and simple hot colloidal synthesis method. Subsequently, the particles were transferred to biological fluid using MSA by ligand exchange process. The functionalized QDs with MSA retained all the original luminescent characteristics and were compatible with biological environments. Moreover, the small molecular weight of MSA functionalized on the QD surface will result in a small hydrodynamic distribution, providing higher chances for excretion from the body after performing their task as tumor biomarkers. Furthermore, we have studied the distribution and clearance of the QDs. Also, we have found that our MSA-functionalized QD formulation has low toxicity, thereby justifying our strategy to functionalize the QD surface with MSA ligands for some aspects of bioimaging applications.

\section{Experimental sections Materials}

Cadmium oxide, selenium, tellurium, zinc acetate, sulfur, oleic acid, high-performance liquid chromatography (HPLC) water, TOP, TOPO, MAA, mercaptopropionic acid, and 
MSA were purchased from Sigma-Adrich Co. All solvents (hexane, toluene, DMSO, and ethanol) were of reagent grade and used without further purification.

\section{Synthesis of $\mathrm{CdTe}_{x} \mathrm{Se}_{1-x} / \mathrm{CdS}$ QDs}

An oleylamine-sulfur solution was generated by mixing $0.1603 \mathrm{~g}$ of sulfur $(1 \mathrm{M})$ in $5 \mathrm{~mL}$ of oleylamine. Se and Te mixture with a molar ratio of 75:25 was mixed with TOP solution. Next, 4 mmol of cadmium oxide was mixed with $8 \mathrm{~g}$ of TOPO and $4 \mathrm{~g}$ of myristic acid. The mixture was heated to $\sim 290^{\circ} \mathrm{C}$ under argon flow, followed by which $1 \mathrm{~mL}$ of TOP:Se:Te solution was introduced into the hot reaction mixture. After that, the temperature was changed to $230^{\circ} \mathrm{C}$, and the mixture was vigorously stirred for 20 minutes, and then $1 \mathrm{~mL}$ of oleylamine-sulfur mixture was slowly introduced into the hot mixture. The final mixture was left undisturbed at $200^{\circ} \mathrm{C}$ for 2 hours. The QDs were purified from the reaction mixture by washing them with ethanol and precipitating them with centrifugation. The QD precipitate can be redispersed in chloroform. Before modifying the QDs surface, the QDs dispersion was filtered using a syringe filter to remove large particulates.

\section{Preparation of MAA-functionalized QDs}

The preparation method of MAA-functionalized QDs aqueous dispersion (MAA-functionalized QDs) was adopted from our previous study. To $2 \mathrm{~mL}$ of $20 \mathrm{mg} / \mathrm{mL}$ of the QDs, $10 \mathrm{~mL}$ of MAA was introduced into the dispersion, and the mixture was stirred for 48 hours. Next, $10 \mathrm{~mL}$ of chloroform was added into the mixture, and the QDs dispersion was centrifuged at 9,000 rpm for 2 hours. The precipitated pellet was washed with chloroform/ethanol solution with repeated centrifugation. Next, $40 \mathrm{mg}$ of MAA-functionalized QDs was dispersed in $5 \mathrm{~mL}$ of phosphate-buffered saline (PBS) solution, and the free MAA molecules were removed by dialysis process against DI water using a dialysis membrane bag with $3.5 \mathrm{kDa}$ cutoff size, and the prepared dispersion was then filtered by employing a $0.45 \mu \mathrm{m}$ syringe filter.

\section{Preparation of MSA-functionalized $\mathrm{CdTe}_{x} \mathrm{Se}_{1-x} / \mathrm{CdS}$ QDs (MSA-functionalized QDs)}

The preparation method of QDs aqueous dispersion was adopted from our previous study. ${ }^{46}$ In this preparation, $4 \mathrm{mmol}$ of MSA was dissolved in $5 \mathrm{~mL}$ of chloroform solution. The mixture was then stirred for 20 minutes, and $2 \mathrm{~mL}$ of concentrated $(\sim 20 \mathrm{mg} / \mathrm{mL})$ QD dispersion was mixed with this solution. A few minutes later, $1 \mathrm{~mL}$ of ammonium hydroxide was introduced into this mixture. This mixture was stirred for more than 24 hours. After that, the QDs were separated from the surfactant solution by adding ethanol to precipitate the QD particles. The precipitate was redispersed in HPLC water, and the prepared dispersion was then filtered by employing a $0.45 \mu \mathrm{m}$ syringe filter. The MSA-functionalized monodispersed QDs (which refer to more than $90 \%$ of the prepared QDs having a particle size of $6 \mathrm{~nm}$ ) displayed excellent colloidal and optical stability, and no precipitation was observed for our prepared formulation within a few weeks of evaluation.

\section{Characterization methods}

The absorption spectra were obtained by using an Agilent $8453 \mathrm{UV}$-vis spectrophotometer (from $300 \mathrm{~nm}$ to $1,100 \mathrm{~nm}$ ). The samples were measured against water as reference. Transmission electron microscopy (TEM) images were obtained using a JEOL model JEM-100CX microscope with an acceleration voltage of $100 \mathrm{kV}$. The specimens were prepared by drop-coating the sample dispersion onto a carbon-coated 300 mesh copper grid. Fluorescence QYs of the QDs in chloroform were measured by comparing the integrated emission from the QDs to Rhodamin 6 dye solutions of matched absorbance. The effective size and size distribution of the QD suspensions were estimated using dynamic light scattering (DLS) particle size analyzer (Brookhaven 90Plus fitted with Avalanche Photodiode (APD) detector using a $656 \mathrm{~nm}$ laser). These solutions were filtered through a $0.45 \mu \mathrm{m}$ syringe filter membrane before the measurement takes place.

\section{Cell viability}

Human pancreatic cancer Panc-1 cells were obtained from American Type Tissue Collection and cultured in Dulbecco's Modified Eagle's Medium (DMEM) supplemented with 10\% fetal bovine serum (Sigma-Aldrich), $1 \mathrm{mM}$ L-glutamine (Sigma-Aldrich), and $100 \mu \mathrm{g} / \mathrm{mL}$ penicillin and streptomycin (Sigma-Aldrich). The day before experiment, 24 culture wells (eight sets, three wells per set) of Panc-1 cells were seeded, and the MTS assay was performed. Briefly, QDs of various concentrations ranging from $25 \mu \mathrm{g} / \mathrm{mL}$ to $350 \mu \mathrm{g} / \mathrm{mL}$ were added to the wells and subsequently incubated with the cells for 24 hours and 48 hours at $37^{\circ} \mathrm{C}$ in a humidified atmosphere with $5 \% \mathrm{CO}_{2}$. After the incubation, $150 \mu \mathrm{L}$ of MTS reagent was added to each well and completely mixed by shaking for 5 minutes. The absorbance at $490 \mathrm{~nm}$ of the mixtures containing formazan that is produced by the cleavage of MTS by dehydrogenases in living cells was measured by using UV-vis spectrophotometer. The percentage of cell 
viability was calculated as the ratio of the absorbance of the QDs-treated well to that of the control well. All data are presented as the mean \pm standard deviation, and the complete assay was repeated three times.

\section{Preparation of pancreatic tumor-bearing mice}

Five- to six-week-old male BALB/c nude mice $(n=4)$ were purchased from the Medical Laboratory Animal Center of Guangdong Province, People's Republic of China and allowed an acclimation period of 1 week. The mice were housed in individually ventilated cages that contained food, water, and bedding, which were sterile. The experiments were performed in accordance with recommendations cited in the Guide for the Care and Use of Laboratory Animals of Laboratory Animal Center of Shenzhen University, Guangdong Province, People's Republic of China (the permit number is SZU-HC-2014-02).

To generate the subcutaneous xenografts, Panc-1 cells were cultured in DMEM to give $85 \%-90 \%$ confluence. The cells was rinsed three times with PBS buffer and harvested with trypsin-ethylenediaminetetraacetic acid (SigmaAldrich). The cells were then centrifuged at 2,000 rpm for 5 minutes, and the supernatant was discarded. The cell pellet was resuspended in fresh medium and counted by a hemocytometer using trypan blue. Then, a 1:1 ratio of cell suspension and Matrigel was made to reach a final cell suspension at density of $3 \times 10^{7}$ Panc- 1 cells $/ \mathrm{mL}$. The mice were injected with $100 \mu \mathrm{L}$ of the cell suspension subcutaneously in the shoulder using a $1 \mathrm{~mL}$ Monoject tuberculin syringe with a $25 \mathrm{~g} \times 5 / 8^{\prime \prime}$ detachable needle. Tumor xenograft formation was monitored every 24-48 hours. Once the tumors reached the appropriate size of $0.5-0.9 \mathrm{~cm}^{2}$, the mice were injected with $150-200 \mu \mathrm{L}$ of functionalized NIR QDs $(50 \mathrm{mg} / \mathrm{kg})$ via tail vein. After injection, mice were anesthetized with isoflurane. The induction concentration was $5 \%$ isoflurane/ $1 \mathrm{~L} \mathrm{O}_{2}$, and the maintenance concentration was $2 \%-3 \%$ isoflurane $/ 1 \mathrm{~L} \mathrm{O}_{2}$. Once the mice were properly anesthetized, they were imaged at indicated time points to monitor the accumulation of QDs in tumors using the Maestro or IVIS Lumina II in vivo optical imaging system. In this study, the scanning wavelength from $500 \mathrm{~nm}$ to $950 \mathrm{~nm}$ was used for the in vivo imaging.

\section{Results and discussion}

\section{Synthesis and characterization of water- dispersible functionalized QDs}

Traditionally, mercapto acids such as MAA, mercaptopyruvic acid, sodium 3-mercapto-1-propanesulfonate, and mercaptopropionic acid are used as ligands to functionalize the surface of QDs for making them water-dispersible and functional, which allows them to conjugate with targeting biomolecules for site-specific delivery. ${ }^{47,48}$ In this study, we have engineered the MSA-functionalized NIR QDs for in vivo imaging applications. The optical and colloidal stability of the MSA-functionalized QDs can be maintained for more than 7-8 weeks at $4^{\circ} \mathrm{C}$. Moreover, with the MSAfunctionalized QDs, one can conjugate targeting molecules with the QDs using the carbodiimide crosslinker chemistry (EDC) for in vitro and in vivo targeted delivery application. More importantly, we have demonstrated that the formulated QDs dispersion can be used for in vivo pancreatic tumor detection and imaging based on passive targeting strategy, without observing any toxic effects to the animals.

The inset illustrates the particle structure of the engineered MSA-functionalized NIR QDs. Basically, the first step involves ligand exchange process of myristic acid-coated QDs with MSA in the aqueous/organic phase. The MSAfunctionalized QDs are dispersible in buffer solution. For bioconjugation, the QDs can be conjugated with proteins, peptides, and antibodies using the straightforward EDC chemistry method.

Figure 1A shows TEM image of water-dispersible NIR QDs. The images demonstrate the high crystallinity of QDs, and the particle average size is $6 \mathrm{~nm}$. Figure $1 \mathrm{~B}$ shows the photoluminescence (PL) spectra from the NIR QDs. The PL spectrum of the QDs shows a band edge emission at $\sim 880 \mathrm{~nm}$. The PL QY of the QDs is estimated to be $\sim 15 \%-20 \%$. The hydrodynamic size and colloidal stability of the QDs dispersed in PBS ( $\mathrm{pH}$ 7.4) were determined using DLS. The result indicates that the prepared MSA-functionalized QD particles have an average hydrodynamic size of $7.5 \mathrm{~nm}$. The effective radius from 0 day to 3 days varied by less than $10 \%$, thus demonstrating their colloidal stability.

\section{In vitro cytotoxicity studies}

Before we applied the QD formulation for in vivo tumor mapping and imaging, in vitro cytotoxicity evaluation of QDs was carefully performed on Panc-1 cells using MTS assay. Figure $1 \mathrm{C}$ shows the cytotoxicity profile for Panc-1 cells treated with different concentrations of the QDs. Overall, the cells maintained $80 \%-90 \%$ viability even after being treated with more than $300 \mu \mathrm{g} / \mathrm{mL}$ MSA-functionalized NIR QDs, for both 24 hours and 48 hours. Besides Panc-1 cells, we have also tested the nanoformulation using other cell lines such as RAW264.7 and UMG87. Similarly, no cytotoxicity effects were observed, and the viability of the 
A

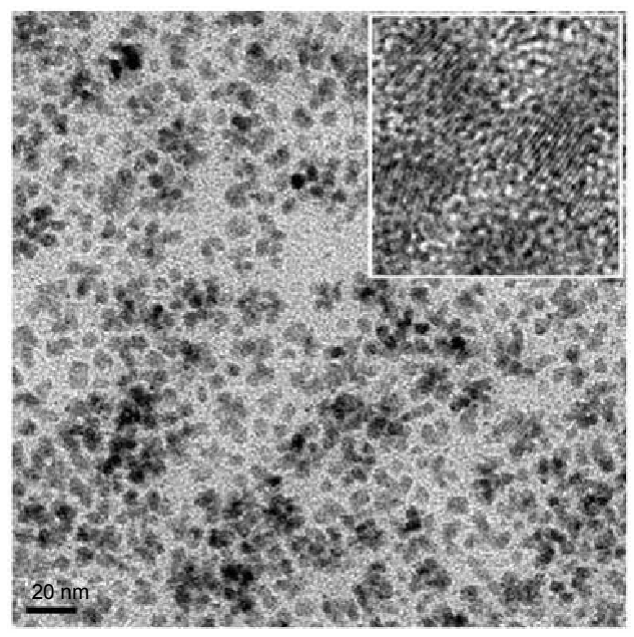

C

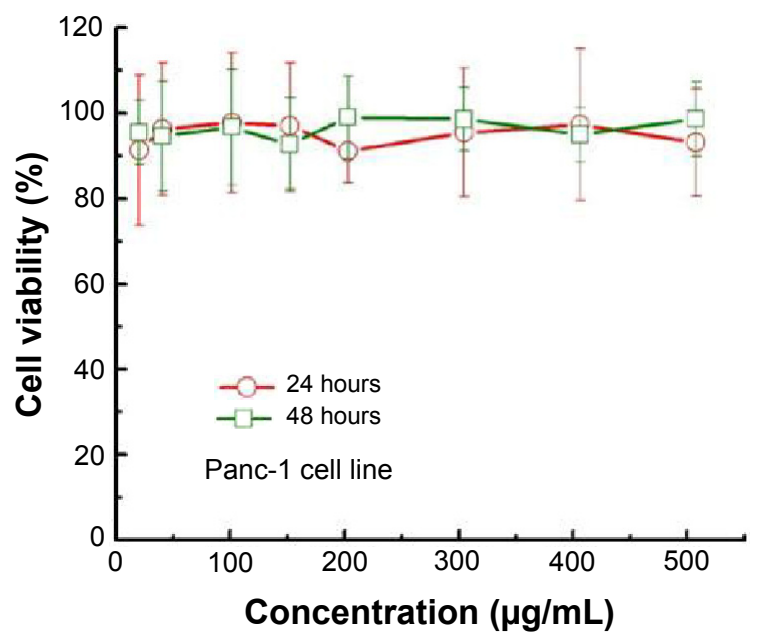

B

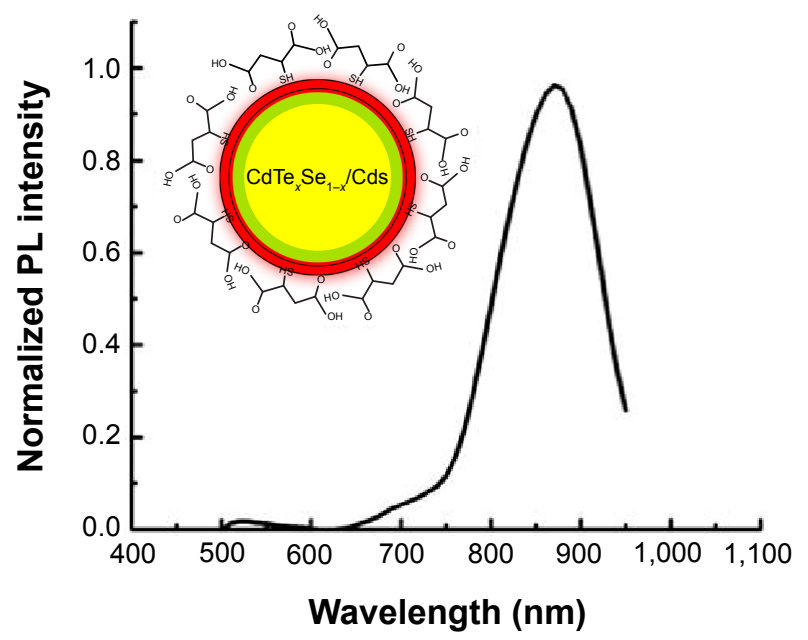

D

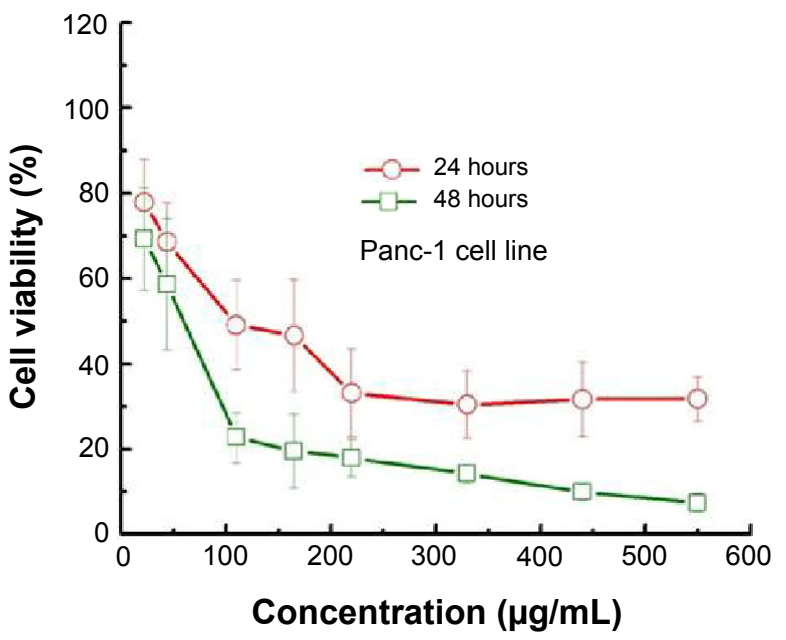

Figure I Characterization and in vitro cytotoxicity study of QDs.

Notes: (A) TEM image of monodispersed MSA-functionalized NIR QDs. The average size of the particles is around $6 \mathrm{~nm}$. (B) Emission spectra of MSA-functionalized QDs. (C) The in vitro cytotoxicity study of MSA functionalized QDs. Panc-I cells were treated with various concentrations of QDs for 24 hours and 48 hours. Values are means $\pm S D, n=3$. (D) The in vitro cytotoxicity study of MAA-functionalized QDs. Panc-I cells were treated with varying concentrations of mercaptoacetic acid-functionalized QDs for 24 hours and 48 hours. Values are means $\pm S D, n=3$.

Abbreviations: TEM, transmission electron microscopy; MSA, mercaptosuccinic acid; MAA, mercaptoacetic acid; NIR, near-infrared; QDs, quantum dots; SD, standard deviation; PL, photoluminescence.

two cell lines was maintained above $80 \%$, with treatment concentration as high as $200 \mu \mathrm{g} / \mathrm{mL}$. This result demonstrated the low toxicity of the QD formulation. In addition to MSA-functionalized QDs, we have also accessed the cytotoxicity of MAA-functionalized QDs formulation with Panc-1 cell line. Figure 1D shows the viability plot of Panc-1 cells treated with various concentrations of MAAfunctionalized NIR QDs. As opposed to MSA-functionalized QDs, the MAA-functionalized QDs were more cytotoxic than the MSA-functionalized QDs, under similar treatment conditions. This finding suggests that the surfactant types play an important role in defining the overall toxicity level of the functionalized QDs.

\section{In vivo tumor imaging studies}

Two approaches have been developed to deliver nanoparticles to tumors, which are active and passive targeting. Passive targeting delivery depends on two unique biological characteristics of the tumor microenvironment. First, the gap between the vascular endothelial cells in tumor tissues is relatively increased, and the capillary walls are leaky, which make it highly permeable to circulating macromolecules in the blood stream. Second, a dysfunctional lymphatic system in the tumor prevents the drainage of accumulated macromolecules and fluids from the tumor interstitial tissues. Therefore, the concentration of nanoparticles (eg, liposomes, micelles) accumulated in the tumor matrix can increase up to 100-150 times 
much more than the ones in the normal tissue. ${ }^{49}$ Such accumulation of macromolecular drugs or nanoparticles in the tumor environment as well as the lack of efficient lymphatic drainage is the so-called enhanced permeability and retention (EPR) effect, and this effect will allow nanoparticles to penetrate through the tumor microvasculature and concentrate themselves in the tumor interstitium. The exudation process of nanoparticles closely relies on the transendothelial channels and the size of endothelial junctions. It was reported that the pore cutoff size of these transport pathways ranged from $400 \mathrm{~nm}$ to $600 \mathrm{~nm}$, and the cutoff size of liposomal extravasation into tumor tissues in vivo was about $400 \mathrm{~nm}$. Therefore, nanoparticles with sizes no more than $200 \mathrm{~nm}$ will extravasate out of tumor microenvironment efficiently. For example, Lee et al reported that the tumor uptake of doxorubicin delivered by dendrimer carrier was ninefold higher than free doxorubicin. ${ }^{50}$ Lee et al reported that glycol chitosan nanoparticles with tumor-targeting ability can serve as a platform delivery carrier in cancer diagnosis and therapy in vivo. ${ }^{51}$ However, these nanoparticles mentioned above cannot be used for real-time monitoring of tumor growth when they are administered into the body. The currently used medical imaging techniques such as computed tomography (CT) and magnetic resonance imaging (MRI) are unable to provide specific dynamic information about the physiological changes of tumor when the cancer patient is treated with drugs formulation. We envision that QDs reported here can be designed, optimized, and integrated with MRI and CT contrast agents to form a targeted multimodal nanoparticle system, which one can use to address the challenges that we face currently. Till date, the hydrodynamic size of functionalized QDs has been reported to be generally in the range from $5 \mathrm{~nm}$ to $60 \mathrm{~nm}$, which suggests that they are suitable to serve as powerful optical probes for EPR-based selective tumor targeting and imaging. On the other hand, active targeting can also be achieved by bioconjugated QDs where their surface is further functionalized with homing agents. In general, QDs can be conjugated with various targeting ligands on their surface, which allows them to specifically recognize and bind to the corresponding receptors that are highly overexpressed in the cancer cells surface. As a result, the nanoparticles can be delivered into the cancer cells with minimum toxicity and off-target side effects.

In this study, we have employed the passive targeting approach to label cancerous area using MSA-functionalized NIR QDs. The mice intravenously injected with the QDs were imaged at different time points using the in vivo imaging system. Figure 2 shows the normalized characterization spectra

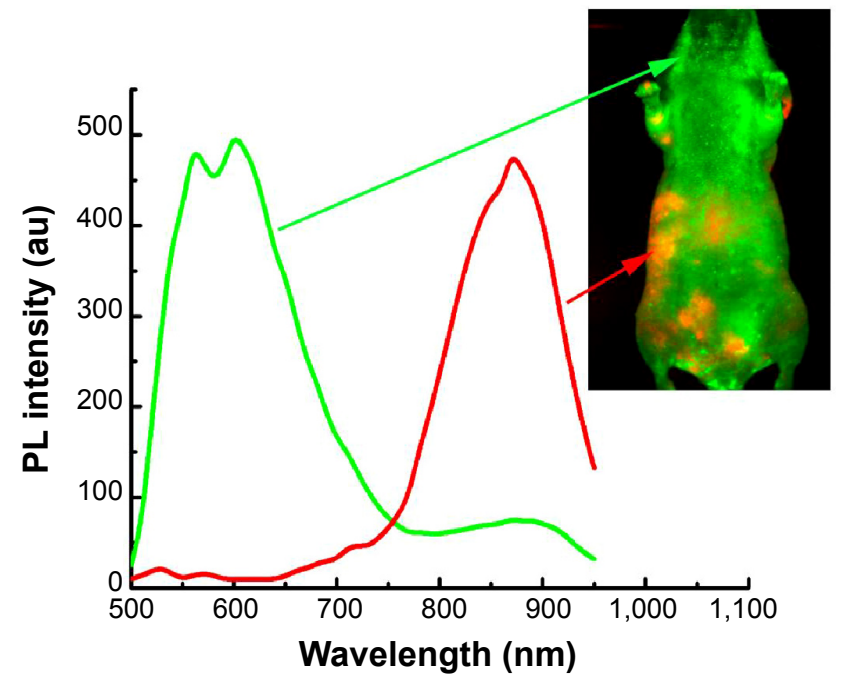

Figure 2 In vivo imaging of pancreatic tumor-bearing mouse injected with MSAfunctionalized NIR QDs.

Notes: The background autofluorescence (from the tissues, skins, and food) is pseudocolored as green, and the QDs signal is pseudocolored as red.

Abbreviations: MSA, mercaptosuccinic acid; NIR, near-infrared; QDs, quantum dots; PL, photoluminescence.

of the background autofluorescence signals and the QD signals from a nude mouse. The background autofluorescence is pseudocolored as green, and QDs signal is pseudocolored as red. Figures 3 and 4 show the luminescence images obtained from the small-animal in vivo imaging system of a pancreatic tumor-bearing mouse post-injected with $\sim 50 \mathrm{mg} / \mathrm{kg}$ of MSA-functionalized QDs.

From Figure 3, one can easily observe a strong luminescence signal from the NIR QDs concentrated in the pancreatic tumor within 30 minutes of injection. During the next 5 hours, a slow decrease in the tumor luminescent intensity can be observed, and no signal was detected at 6 hours postinjection. The treated mice were further imaged for another 200 hours to examine the distribution and clearance of NIR QDs. No mortality was observed in the mice group, demonstrating the low toxicity of the functionalized NIR QDs. It is worth mentioning that fluorescent signal of QDs from the upper neck of the injected mouse was also obtained, which is probably because the cancer cells have spread excessively from the primary site into surrounding tissue in vivo. Once the in vivo whole-body imaging was completed, the mice were sacrificed, and the major organs (the heart, liver, spleen, lung, and kidney) were dissected and then imaged by the in vivo optical imaging system. As shown in Figure 5, the luminescence signals were detected in the spleen and liver, which indicated that QDs were taken up by these organs and accumulated in these tissues. In addition, minimal QD signal was detected in the other organs such as the kidney, lung, 


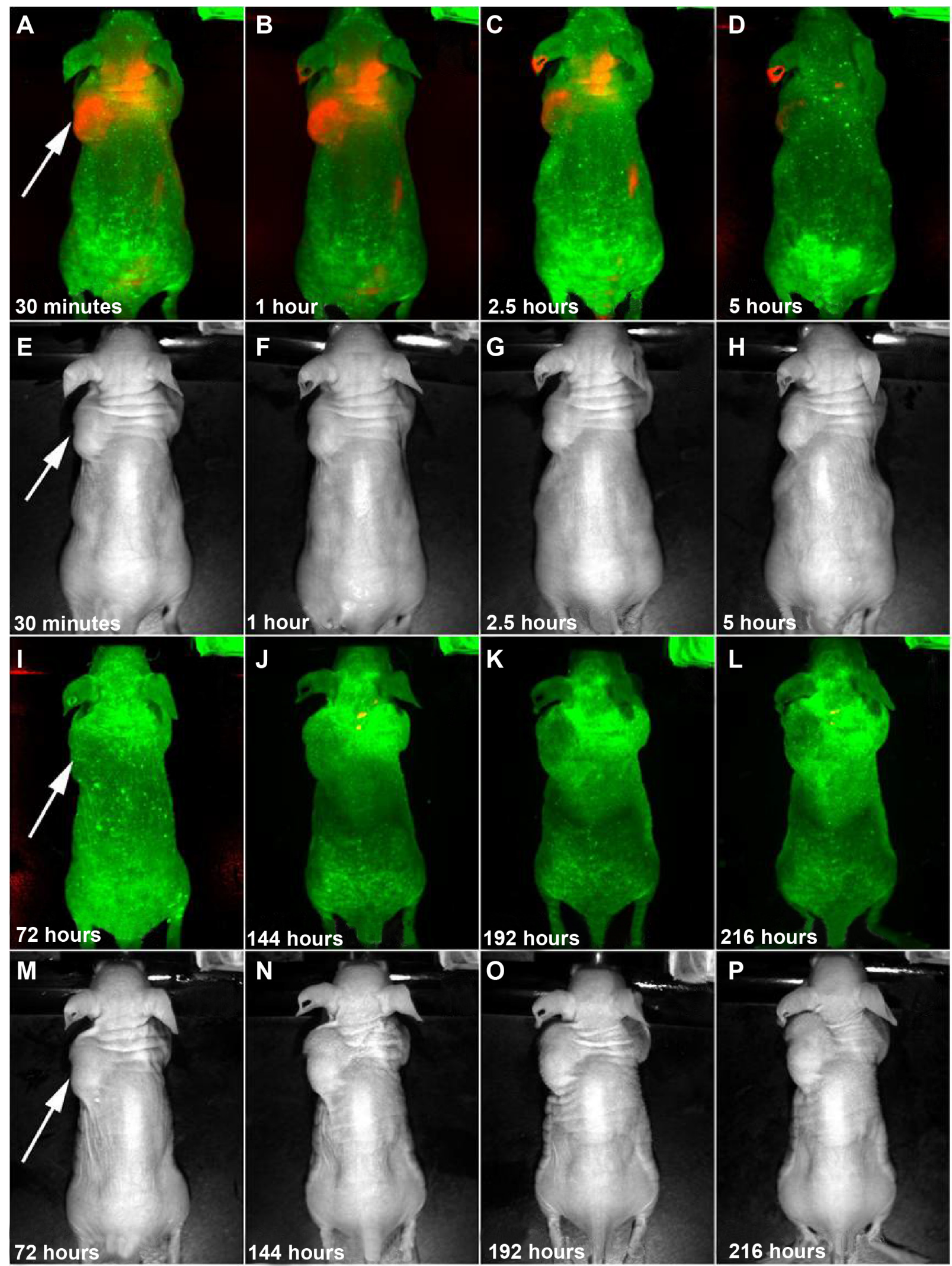

Figure 3 Time-dependent in vivo luminescence imaging of Panc-I tumor-bearing mice (left shoulder, tumor pointed by white arrows) injected with 50 mg/kg of MSAfunctionalized NIR QDs, with background in green and the QD signals in red.

Notes: All images were acquired under the same experimental conditions. Transmission images in (E-H) and (M-P) correspond to the luminescence images in (A-D) and (I-L), respectively.

Abbreviations: MSA, mercaptosuccinic acid; NIR, near-infrared; QDs, quantum dots. 

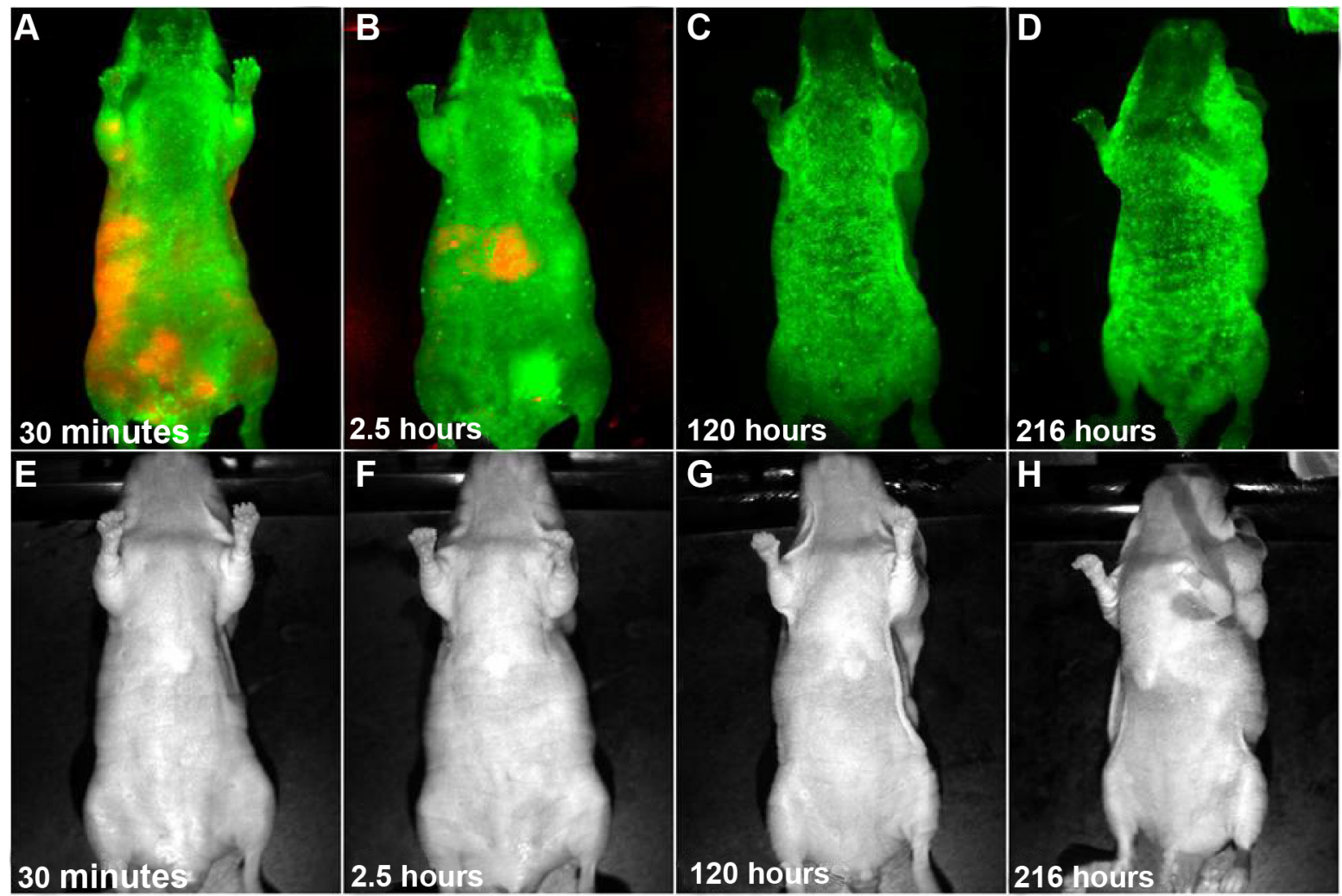

30 minutes
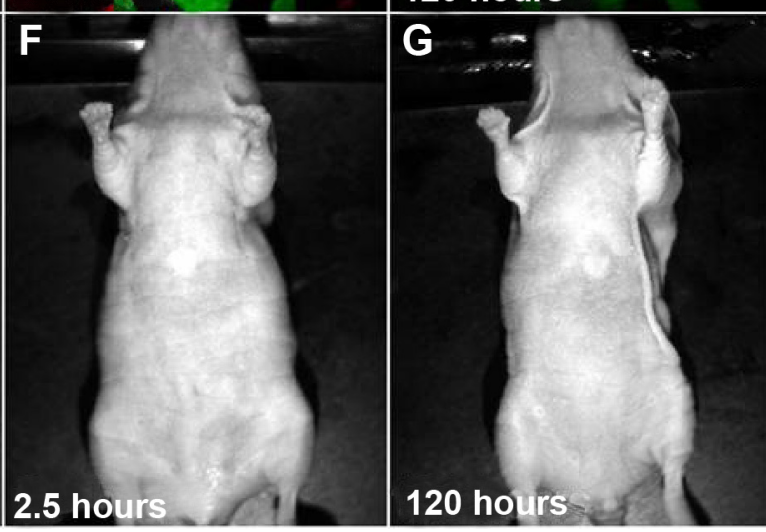

Figure 4 Lateral view of in vivo luminescence imaging of Panc-I tumor-bearing mice (left shoulder) injected with $\sim 50 \mathrm{mg} / \mathrm{kg}$ of MSA-functionalized QDs.

Note: All images were acquired under the same experimental conditions. Transmission images in (E-H) correspond to the luminescence images in (A-D), respectively. Abbreviations: MSA, mercaptosuccinic acid; QDs, quantum dots.

and heart. The biodistribution of these QDs in major organs is consistent with the previous reported studies. ${ }^{49}$

For QDs that do not excrete through renal clearance (with particles size less than $6 \mathrm{~nm}$ ), the only other major route of excretion from the body is through the liver, via the bile, and feces. ${ }^{18}$ To date, there are a few general

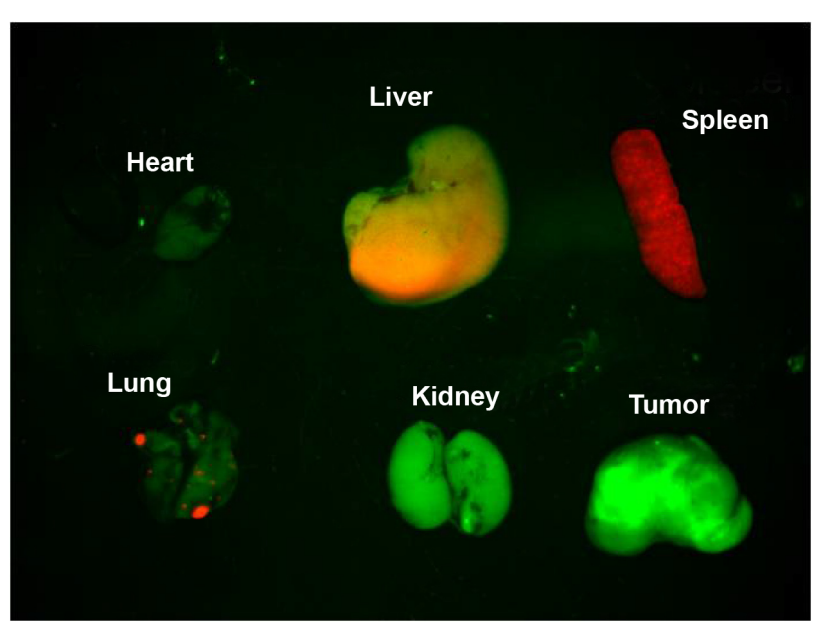

Figure 5 Luminescent images of major organs and tumor from Panc-I tumorbearing mouse injected with NIR QDs.

Notes: The autofluorescence from organs is coded green, and the unmixed QD signal is coded red. Prominent uptake in the liver and spleen was visible. Abbreviations: NIR, near-infrared; QDs, quantum dots. observations with liver excretion for functionalized QDs. ${ }^{49}$ First, the liver is "programmed" to filter and remove foreign particles with hydrodynamic diameter $>50 \mathrm{~nm}$. The use of special coatings such as PEG on the QD surface to prevent the removal by the reticuloendothelial system (RES) (the liver, spleen, and bone marrow) has been reported. These PEG coatings will generally increase the hydrodynamic size of the QDs. PEGylation on nanoparticle surface will also increase blood half-life. However, it also slows the excretion rate of functionalized QDs from the body. Second, excretion of QDs into bile is a slow process. But, it is suggested that as long as the particles do not degrade during the "slow" excretion process, minimal or no damage will occur in the body. Third, long-term retention of the leftover QDs in the RES might lead to a large area under the exposure-time curve, and this will potentially increase the chances of in vivo nanoparticle toxicity. To overcome this issue, one can coat thicker inorganic shell (eg, $\mathrm{ZnS}$ shell) on the QDs surface to prevent them from breaking down during the liver excretion process. An alternative approach to minimize the chances of QD degradation in the liver and spleen is to passivate their surface with long-lasting biocompatible polymer layer, which will enhance their overall stability. 
Several groups have investigated the distribution and clearance of QDs in vivo. ${ }^{52,53}$ From their reports, some common features were observed for the biodistribution of functionalized QDs. Previously, Gao et al have reported that $15 \mathrm{~nm} \mathrm{CdSe} / \mathrm{ZnS}$ QDs were prepared, and the particle surfaces were carefully functionalized with block copolymer layer and targeting molecules for specific tumor mapping and imaging. ${ }^{44}$ They have found that appreciable amounts of QDs were accumulated in the lung, liver, and spleen, even when the surface of QDs was linked with PEG molecules with molecular weight as high as 2,000 Da. Nevertheless, the authors were still able to use these optical probes to label the tumor sites. Recently, Cai et al reported the use of targeted $\mathrm{CdTe} / \mathrm{ZnS}$ QDs emitting at $705 \mathrm{~nm}$ with hydrodynamic size of $20 \mathrm{~nm}$ for NIR imaging of tumor in vivo. ${ }^{45}$ Within 30 minutes of injection, major fraction of QDs was found in the liver and spleen, based on their noninvasive whole-body optical imaging system. Though the exact biodistribution of the nanoparticles was not reported in their study, the authors have clearly mentioned that it is critical to manipulate the surface coating of QDs for enhancing nanoparticle removal from the body. Chen et al have prepared MAA-functionalized $\mathrm{CdSe} / \mathrm{ZnS}$ QDs as probes for liver cancer detection and imaging. ${ }^{54}$ Similarly, the authors have observed that the QD probes were mainly distributed in the liver, spleen, and kidney. The hydrodynamic size was not reported in this study. No direct comparison can be made with our formulation. However, the authors have claimed that their formulation is nontoxic by performing animal body weight evaluation and blood analysis on the treated animals. All these results suggested that these functionalized QDs can be further improved for specific in vivo applications. Further studies are needed to reengineer the current QDs so that they can efficiently and safely excrete from the body.

\section{Conclusion}

In summary, we have reported the preparation of MSAfunctionalized NIR QDs that retain small hydrodynamic size, high QY, and surface functionality; the results provide a unique platform for potential cancer diagnostic and therapeutic applications. These MSA-functionalized QDs can be readily used for targeting and imaging tumors in live animals without causing any ill effects to the animals. By using the whole-body fluorescent imaging technique, we have demonstrated that the functionalized QDs accumulated in the tumor matrix by EPR effect. The accumulation of the QDs in the tumor was observed to last for more than 2.5 hours. Some fractions of QDs were found to remain in the spleen and liver. Further studies are needed to confirm whether the sequestered QDs will be slowly removed by liver excretion. These findings not only offer useful information for designing targeted cancer nano-imaging but also are useful in the design and development of parameters of QD formulations for early pancreatic cancer detection and image-guided surgery applications.

\section{Acknowledgments}

This work was supported by the grants from National Natural Science Foundation of China (81301318), Shenzhen Basic Research and Key Laboratory Project (JCYJ20120613170218654, JC201005280391 A, JCYJ20140418182819164, ZDSY20130329101130496), the Singapore Ministry of Education (Grants Tier 2 MOE2010T2-2-010 [M4020020.040 ARC2/11], Tier 1 M4010360.040 RG29/10), NTU-NHG Innovation Collaboration Grant (number M4061202.040), and A*STAR Science and Engineering Research Council (number M4070176.040).

\section{Disclosure}

No conflict of interest exists in the submission of this manuscript and none declared.

\section{References}

1. Bruchez M, Moronne M, Gin P, Weiss S, Alivisatos AP. Semiconductor nanocrystals as fluorescent biological labels. Science. 1998; 281(5385):2013-2016.

2. Petryayeva E, Algar WR, Medintz IL. Quantum dots in bioanalysis: a review of applications across various platforms for fluorescence spectroscopy and imaging. Appl Spectrosc. 2013;67(3):215-252.

3. Yong KT, Sahoo Y, Zeng H, Swihart MT, Minter JR, Prasad PN. Formation of ZnTe nanowires by oriented attachment. Chem Mater. 2007;19(17):4108-4110.

4. Farkhani SM, Valizadeh A. Review: three synthesis methods of $\mathrm{CdX}$ (X $=\mathrm{Se}, \mathrm{S}$ or Te) quantum dots. IET Nanobiotechnol. 2014;8(2):59-76.

5. Park JH, Gu L, von Maltzahn G, Ruoslahti E, Bhatia SN, Sailor MJ Biodegradable luminescent porous silicon nanoparticles for in vivo applications. Nat Mater. 2009;8(4):331-336.

6. Wang YQ, Chen LX. Quantum dots, lighting up the research and development of nanomedicine. Nanomedicine. 2011;7(4):385-402.

7. Lin GM, Yin F, Yong KT. The future of quantum dots in drug discovery. Expert Opin Drug Discov. 2014;9(9):991-994.

8. Allen PM, Bawendi MG. Ternary I-III-VI quantum dots luminescent in the red to near-infrared. J Am Chem Soc. 2008;130(29):9240-9241.

9. Chan WCW, Nie SM. Quantum dot bioconjugates for ultrasensitive nonisotopic detection. Science. 1998;281(5385):2016-2018.

10. Cheki M, Moslehi M, Assadi M. Marvelous applications of quantum dots. Eur Rev Med Pharmacol Sci. 2013;17(9):1141-1148.

11. Altinoglu EI, Adair JH. Near infrared imaging with nanoparticles. Wiley Interdiscip Rev Nanomed Nanobiotechnol. 2010;2(5):461-477.

12. Dubertret B, Skourides P, Norris DJ, Noireaux V, Brivanlou AH, Libchaber A. In vivo imaging of quantum dots encapsulated in phospholipid micelles. Science. 2002;298(5599):1759-1762.

13. Erogbogbo F, Yong KT, Roy I, Xu G, Prasad PN, Swihart MT. Biocompatible luminescent silicon quantum dots for imaging of cancer cells. ACS Nano. 2008;2(5):873-878.

14. Mulder WJ, Strijkers GJ, Nicolay K, Griffioen AW. Quantum dots for multimodal molecular imaging of angiogenesis. Angiogenesis. 2010;13(2):131-134. 
15. Yong KT, Qian J, Roy I, et al. Quantum rod bioconjugates as targeted probes for confocal and two-photon fluorescence imaging of cancer cells. Nano Lett. 2007;7(3):761-765.

16. Yong KT, Sahoo Y, Swihart MT, Schneeberger PM, Prasad PN. Templated synthesis of gold nanorods (NRs): the effects of cosurfactants and electrolytes on the shape and optical properties. Top Catal. 2008; 47(1-2):49-60.

17. Ma QA, Su XG. Near-infrared quantum dots: synthesis, functionalization and analytical applications. Analyst. 2010;135(8):1867-1877.

18. Yong KT, Roy I, Ding H, Bergey EJ, Prasad PN. Biocompatible nearinfrared quantum dots as ultrasensitive probes for long-term in vivo imaging applications. Small. 2009;5(17):1997-2004.

19. Sharma P, Brown S, Walter G, Santra S, Moudgil B. Nanoparticles for bioimaging. Adv Colloid Interface Sci. 2006;123:471-485.

20. Zhang Y, Mi L, Wang PN, et al. Photoluminescence decay dynamics of thiol-capped CdTe quantum dots in living cells under microexcitation. Small. 2008;4(6):777-780.

21. Ko MH, Kim S, Kang WJ, et al. In vitro derby imaging of cancer biomarkers using quantum dots. Small. 2009;5(10):1207-1212.

22. Michalet X, Pinaud FF, Bentolila LA, et al. Quantum dots for live cells, in vivo imaging, and diagnostics. Science. 2005;307(5709):538-544.

23. Azzazy HME, Mansour MMH, Kazinierczak SC. From diagnostics to therapy: prospects of quantum dots. Clin Biochem. 2007;40(13-14): 917-927.

24. He XX, Wang KM, Cheng Z. In vivo near-infrared fluorescence imaging of cancer with nanoparticle-based probes. Wiley Interdiscip Rev Nanomed Nanobiotechnol. 2010;2(4):349-366.

25. Li C, Zhang Y, Wang M, et al. In vivo real-time visualization of tissue blood flow and angiogenesis using Ag2S quantum dots in the NIR-II window. Biomaterials. 2014;35(1):393-400.

26. Shao D, Li J, Xiao X, et al. Real-time visualizing and tracing of HSV-TK/GCV suicide gene therapy by near-infrared fluorescent quantum dots. ACS Appl Mater Interfaces. 2014;6(14):11082-11090.

27. Katari S, Wallack M, Huebschman M, Pantano P, Garner H. Fabrication and evaluation of a near-infrared hyperspectral imaging system. J Microsc. 2009;236(1):11-17.

28. Lee $\mathrm{CH}$, Cheng $\mathrm{SH}$, Wang YJ, et al. Near-infrared mesoporous silica nanoparticles for optical imaging: characterization and in vivo biodistribution. Adv Funct Mater. 2009;19(2):215-222.

29. Jin T, Yoshioka Y, Fujii F, Komai Y, Seki J, Seiyama A. Gd3+functionalized near-infrared quantum dots for in vivo dual modal (fluorescence/magnetic resonance) imaging. Chem Commun (Camb). 2008;44:5764-5766.

30. Hezinger AF, Tessmar J, Gopferich A. Polymer coating of quantum dots - a powerful tool toward diagnostics and sensorics. Eur J Pharm Biopharm. 2008;68(1):138-152.

31. Liu L, Yong KT, Roy I, et al. Bioconjugated pluronic triblock-copolymer micelle-encapsulated quantum dots for targeted imaging of cancer: in vitro and in vivo studies. Theranostics. 2012;2(7):705-713.

32. Li X, Chen N, Su Y, et al. Autophagy-sensitized cytotoxicity of quantum dots in PC12 cells. Adv Healthc Mater. 2014;3(3):354-359.

33. Soenen SJ, Manshian BB, Aubert T, et al. Cytotoxicity of cadmiumfree quantum dots and their use in cell bioimaging. Chem Res Toxicol. 2014;27(6):1050-1059.

34. Tsoi KM, Dai Q, Alman BA, Chan WCW. Are quantum dots toxic? Exploring the discrepancy between cell culture and animal studies. Acc Chem Res. 2013;46(3):662-671.

35. Jiang S, Win KY, Liu SH, Teng CP, Zheng YG, Han MY. Surfacefunctionalized nanoparticles for biosensing and imaging-guided therapeutics. Nanoscale. 2013;5(8):3127-3148.
36. Speranskaya ES, Beloglazova NV, Lenain P, et al. Polymer-coated fluorescent $\mathrm{CdSe}-$ based quantum dots for application in immunoassay. Biosens Bioelectron. 2014;53:225-231.

37. Smith AM, Duan HW, Mohs AM, Nie SM. Bioconjugated quantum dots for in vivo molecular and cellular imaging. Adv Drug Deliv Rev. 2008;60(11):1226-1240.

38. Song YC, Feng D, Shi W, Li XH, Ma HM. Parallel comparative studies on the toxic effects of unmodified CdTe quantum dots, gold nanoparticles, and carbon nanodots on live cells Cas well as green gram sprouts. Talanta. 2013;116:237-244.

39. Wu TS, Tang M. Toxicity of quantum dots on respiratory system. Inhal Toxicol. 2014;26(2):128-139.

40. Chang E, Thekkek N, Yu WW, Colvin VL, Drezek R. Evaluation of quantum dot cytotoxicity based on intracellular uptake. Small. 2006;2(12):1412-1417.

41. Subramaniam P, Lee SJ, Shah S, Patel S, Starovoytov V, Lee KB. Generation of a library of non-toxic quantum dots for cellular imaging and siRNA delivery. Adv Mater. 2012;24(29):4014-4019.

42. Choi HS, Liu W, Misra P, et al. Renal clearance of quantum dots. Nat Biotechnol. 2007;25(10):1165-1170.

43. Qian X, Peng XH, Ansari DO, et al. In vivo tumor targeting and spectroscopic detection with surface-enhanced Raman nanoparticle tags. Nat Biotechnol. 2008;26(1):83-90.

44. Gao XH, Cui YY, Levenson RM, Chung LWK, Nie SM. In vivo cancer targeting and imaging with semiconductor quantum dots. Nat Biotechnol. 2004;22(8):969-976.

45. Cai W, Shin DW, Chen K, et al. Peptide-labeled near-infrared quantum dots for imaging tumor vasculature in living subjects. Nano Lett. 2006;6(4):669-676.

46. Bharali DJ, Lucey DW, Jayakumar H, Pudavar HE, Prasad PN. Folate-receptor-mediated delivery of InP quantum dots for bioimaging using confocal and two-photon microscopy.J Am Chem Soc. 2005;127(32): 11364-11371.

47. Rogach AL, Franzl T, Klar TA, et al. Aqueous synthesis of thiol-capped CdTe nanocrystals: state-of-the-art. J Phys Chem C. 2007;111(40):14628-14637.

48. Zhang H, Wang DY, Yang B, Mohwald H. Manipulation of aqueous growth of CdTe nanocrystals to fabricate colloidally stable one-dimensional nanostructures. J Am Chem Soc. 2006;128(31):10171-10180.

49. Yong KT, Roy I, Swihart MT, Prasad PN. Multifunctional nanoparticles as biocompatible targeted probes for human cancer diagnosis and therapy. J Mater Chem. 2009;19(27):4655-4672.

50. Lee CC, Gillies ER, Fox ME, et al. A single dose of doxorubicinfunctionalized bow-tie dendrimer cures mice bearing C-26 colon carcinomas. Proc Natl Acad Sci US A. 2006;103(45):16649-16654.

51. Lee SJ, Min HS, Ku SH, et al. Tumor-targeting glycol chitosan nanoparticles as a platform delivery carrier in cancer diagnosis and therapy. Nanomedicine. 2014;9(11):1697-1713.

52. Santra S, Yang H, Stanley JT, et al. Rapid and effective labeling of brain tissue using TAT-conjugated $\mathrm{CdS}$ : $\mathrm{Mn} / \mathrm{ZnS}$ quantum dots. Chem Commun (Camb). 2005;25:3144-3146.

53. Nagy A, Steinbruck A, Gao J, Doggett N, Hollingsworth JA, Iyer R. Comprehensive analysis of the effects of CdSe quantum dot size, surface charge, and functionalization on primary human lung cells. ACS Nano. 2012;6(6):4748-4762.

54. Chen LD, Liu J, Yu XF, et al.The biocompatibility of quantum dot probes used for the targeted imaging of hepatocellular carcinoma metastasis. Biomaterials. 2008;29(31):4170-4176. 
International Journal of Nanomedicine

Dovepress

\section{Publish your work in this journal}

The International Journal of Nanomedicine is an international, peerreviewed journal focusing on the application of nanotechnology in diagnostics, therapeutics, and drug delivery systems throughout the biomedical field. This journal is indexed on PubMed Central, MedLine, CAS, SciSearch ${ }^{\circledR}$, Current Contents ${ }^{\circledR} /$ Clinical Medicine,
Journal Citation Reports/Science Edition, EMBase, Scopus and the Elsevier Bibliographic databases. The manuscript management system is completely online and includes a very quick and fair peer-review system, which is all easy to use. Visit http://www.dovepress.com/ testimonials.php to read real quotes from published authors.

Submit your manuscript here: http://www.dovepress.com/international-journal-of-nanomedicine-journal 